superior maxillary of the left side, the lohe of the brain corresponding to the injury, was disorganised, and infiltrated with pus. The pericranium was highly ecchymosed; but the dura mater and brain, not in the vicinity of the fracture, were quite in a natural state.

\section{RESTORATION OF LOST PARTS.}

By M. Lisfranc.

Destruction of the whole of the Lower Lip and Part of the Cheek; Restoration by a Flap of Integument from the Neck.

THE patient, a man 55 years of age, of sanguine temperament, was admitted into the Maison de Sante, Rue Marbouf, on the 20 th of June, 1840.

About ten years back he observed a pimple on the middle of his chin, which was removed by incision and cauterisation with arsenical paste. At the end of three years two excrescences, similar to the first, made their appearance near the angles of the month. They yielded to the same kind of treatment, but were followed by suspiciouslooking ulcerations, attended with lancinating pains, and showing a disposition to spread. The patient had never suffered from syphilis, and none of his family were affected with cancer.

The cause of the disease is probably the habit of smoking, and drinking alcoholic liquors, inasmuch as several persons living in the same district with himself, and addicted to the same practice, are suffering in a similar manner. It is, moreover, to be observed, that the patient has exposed the lip, in its ulcerated state, to the most rigorous weather, without using any protective covering. The ulcerations have also been increased by the mistimed application of irritating remedies. Under these circumstances the prospect of cure, from operative procedure, offers every hope of success.

At the present time his constitution is good, the vital organs perform their functions regularly, and he regards the anticipation of the operation with fortitude. The whole of the lower lip, as far as the inferior border of the lower jaw, is gone; the bone is not cancerous, but seems somewhat hypertrophied; the glands in the neck are not affected.

June 28. M. Lisfranc removed, by a semilunar and two horizontal incisions, the whole of the diseased edges. The cut surface was very vascular, and required the ligature or torsion of ten small arteries. The operator then made an incision along the midule line of the neck to the thyroid cartilage, and dissected back a flap at either side. Finding the bone more affected with the disease than was at first thought, he removed several of the teeth, and cut off the alveolar process with the bone-nippers. The bone was somewhat softened, and presented a slaty hue. He then cut off the projecting angle of the symphisis with a saw, to remove the danger of its projection between the flaps, in the progress of the cure. The flaps were now brought over the bone, and were found to supply perfectly the place of the lost lip. The edges of the flaps were brought together by meaus of fourteen pins, armed with the twisted suture.

29. A blush of erysipelas on the left side of the neck. Apply hog's lard to the inflamed surface. Venesection.

July 1. M. Lisfranc removed all the pins, with the exception of those which held the angles of the wound. The erysipelas has extended to the right side. Continue the hog's lard upon compresses.

2. The rest of the pins removed; union by the first intention is complete thronghout.

4. Two excavations have appeared at the angles of the mouth, from which a quantity of pus is pressed out several times during the day.

5. M. Lisfranc opened a small abscess situated at about an inch below the chin; he also made two counter-openings for the purulent excavations above described.

7. A counter-opening was made externally to each external jugalar vein, to relieve the cellular tissue of the neck of an extensive purulent infiltration.

10. In pressing upon the side of the neck, a kind of cord was felt beneath the integument, which was taken at first for the omo. hyoid musele. On examining the counteropening, some dead cellular tissue was seen, which, on being removed with the forceps, was found to be part of the ronnded mass mistaken for the muscle. From this moment the erysipelas wholly subsided, and the purulent excavations became filled up with granulations. The alveolar border was touched for some time with the nitrate of silver, and protected from the irritation of the saliva by a compress, covered with an ointment of acetate of lead. The patient quitted the Maison de Santé with little deformity existing, and perfectly cured.Gazette de Hôpitaux, Aug. 20.

\section{APOTHECARIES IN RUSSIA.}

But, in this analysis of the more respectable part of the population of a Russian town, we have omitted one conspicuous person-the apothecary. $\mathrm{He}$ is always among the wealthiest in the place. None can sell drugs without a patent; and as only one or two in a provincial town, willing to gain their bread in this way, have influence enough to obtain the emperor's permission, there is but little opposition in the trade. Nothing is paid for the patent, so 